\title{
Panoramic View of the Heat Flux Inside an Insulated Vehicle by Infrared Thermography
}

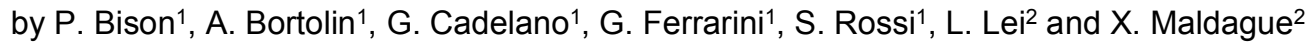 \\ ${ }^{1}$ CNR-ITC, C.so Stati Uniti 4, 35127 Padova, Italy \\ ${ }^{2}$ ECE Dept., Universitè Laval, G1V0A6, Quèbec, Canada
}

\begin{abstract}
An IR camera is mounted on a pan-tilt head and automatically driven to map the temperature of the inner walls of the insulated box on a refrigerated vehicle. The vehicle is introduced in a test station where special conditions are applied, in such a way to maintain an inner-outer temperature difference of around $25^{\circ} \mathrm{C}$. The objective of this work is that of complementing the above qualitative results obtained by IR thermography, with an evaluation of the heat flux flowing through the wall, possibly at the pixel level.
\end{abstract}

\section{Introduction}

Nowadays the correct transport of perishable foodstuffs in the refrigerated vehicles, especially for dairy products, meat and frozen foods, is going with the necessity of energy saving due to the ever increasing cost of energy. So it is essential that the refrigerated vehicles are equipped with a suitable thermal insulation in order to save energy maintaining at the same time an appropriate conservation of the foodstuffs. There exits some agreements of the thermal insulation tests which ensures the suitability for the transport of food in refrigerated conditions-ATP. "The Agreement on the Transport of Perishable foodstuffs"[1], which establishes standards for the international transport of perishable food between the states that ratify the treaty.

CNR-ITC owns a consolidated experience in the measurement of heat transfer coefficient $\mathrm{K}$ applied to refrigerated vehicles ([2],[3],[4],[5],[6]). It is also responsible for the certification of these vehicles throughout Italy.

In summary, the method for measuring and checking the insulation of the equipment during the ATP test [3], is that of measuring the overall coefficient of heat transfer $K$, defined as:

$$
K=\frac{W}{S \cdot \Delta \theta}
$$

where $W$ is the power necessary to maintain a steady temperature difference $\Delta \theta$ between the mean internal and external air temperature of the box and $S$ is the mean surface of the box, given by the geometric mean of the inside and outside surface areas:

$$
S=\sqrt{S_{i} \cdot S_{e}}
$$

The ATP standard test is a procedure to measure the status of insulated vehicles with a global approach. Its robustness has been well demonstrated. On the other hand, some local defects in the structure, such as thermal bridges, air leakages or zones of anomalous aging, even though taken into account globally, cannot be localized in the structure. IR thermography can be particularly helpful to these issues. In fact, all the defects mentioned above lead to a variation of the heat flux and temperature on the surface of the box ([8],[9]). Therefore the local heat flux map obtained by Infrared thermography could give a straightforward visualization of the structure, and also a local evaluation of the transmittance.

This work consists of the image processing applied to an insulated box on a refrigerated vehicle. In practice, the experimentation will be performed to map the heat flux on one of its internal surface by Infrared thermography. When considering all the surfaces a panoramic view of the heat flux will be composed.

\section{The heat transfer model}

In what follows we describe a simplified model of the heat transfer from inside the equipment under test and the outside. In order to meet the requirements of ATP standard a heater is working inside the insulated vehicle. When steady conditions are reached it delivers a power $W$ in order to maintain a steady air temperature $\theta_{i}$ inside the box. Because the external temperature is lower than the internal one, heat flows from inside to the outside of the box where a steady air temperature is maintained at the level of $\theta_{e}$. Heat is transferred by convection from the hot inside air to the internal wall, by conduction through the solid wall and again by convection from the external wall to the cold outside air according to the scheme of Figure 1. In the hypotheses that the heat flux is essentially 1D, $h_{e}$ and $h_{i}$ are respectively the effective heat 
exchange coefficients external and internal. $\theta_{w e}$ and $\theta_{w i}$ are the average temperature of the internal and external walls, while $\lambda$ is the thermal conductivity of the wall and $/$ its thickness. Of course, the central thermal resistance of Figure 1 is the sum of the individual thermal resistance given by the different layers of the insulated wall. In many cases three layers composes the wall of an insulated box mounted on a truck: two internal-external skins made by polyester-fiberglass and a core made by high density polyurethane foam.

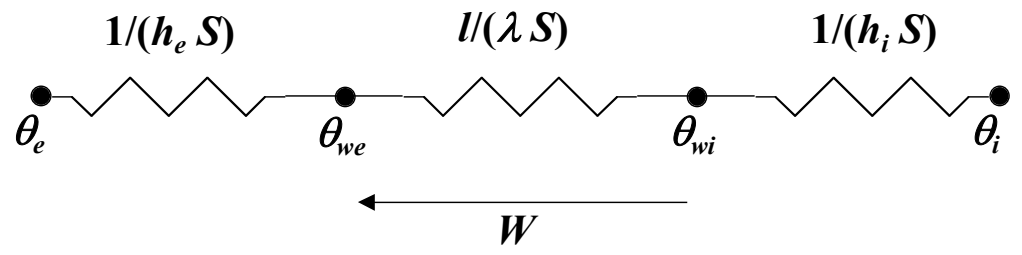

Fig. 1. Overall thermal behaviour of the container under test represented as an analogy of an electric circuit.

What is seen by looking with thermography at the internal wall of the insulated container is a local variation of temperature that is mainly due to a reduction of insulation. For instance, where two insulation panels are jointed a thermal bridge is given by the higher thermal conductivity of the polyester resin than the polyurethane foam. It means that a local variation of thermal conductivity leads to a local increase of the heat flux with a consequent variation of the local internal and external wall temperature. Thanks to the possibility given by thermography of mapping the external temperature of the insulated box it is more convenient to turn from a global view to a local view of the heat transfer. Specifically, by measuring in a reference zone of the external wall the specific heat flux by a thermal flux meter, and by looking at the wall temperature in the proximity of the flux meter and by measuring the air temperature the following local equation can be drawn:

$$
q_{r}=\frac{\theta_{w i}\left(x_{r}, y_{r}\right)-\theta_{i}}{1 / h_{i}}
$$

where $q_{r}$ is the specific heat flux measured at the reference point by the heat flux meter and $\theta_{w e}\left(x_{r}, y_{r}\right)$ is the temperature measured by thermography at the coordinate of the reference point. The assumption that the heat exchange coefficient $h_{i}$ does not vary significantly with the position permits to take the temperature and flux measurements in the reference point as a pivot, chosen after a preliminary thermographic scanning. The heat flux map may be determined basing on the temperature map obtained by thermography according to the following equation:

$$
q(x, y)=q_{r} \frac{\theta(x, y)-\theta_{i}}{\theta_{w i}\left(x_{r}, y_{r}\right)-\theta_{i}}=\frac{q_{r}}{\Delta \theta_{r}} \Delta \theta_{x, y}+q_{r}=h_{i} \Delta \theta_{x, y}+q_{r}
$$

where $\Delta \theta_{x, y}=\left(\theta(x, y)-\theta_{i}\right)$ and $\Delta \theta_{r}=\theta_{w i}\left(x_{r}, y_{r}\right)-\theta_{i}$.

Equation 4 gives $q(x, y)$ as a linear relation depending on $\Delta \theta_{x, y}$, with intercept $q_{r}$ and slope $h_{i}$. It shows that the mapping of heat flux depends linearly on the measurement of the temperature difference between any point of the surface and the reference point (nearby the position of the heat flux meter). The mapping of temperature difference is one of the points of strength of IR thermography that, in high quality equipment, can reach a NETD (Noise Equivalent Temperature Difference) as low as $20 \mathrm{mK}$. In case of surface temperature variation statistically distributed around the $\theta_{w i}\left(x_{r}, y_{r}\right)$ value, $\Delta \theta_{x, y}$ will be very close to zero in the average and we expect to obtain an average value of the heat flux very close to the reference one $q_{r}$. In such a case the uncertainty of the average value of $q(x, y)$ depends essentially on the accuracy of the $q_{r}$ that we can estimate in $\pm 5 \%$. When thermal bridges are present on the structure, $\Delta \theta_{x, y}$ becomes negative in the average and the average value of $q(x, y)$ is expected to over $q_{r}$. In this case it is important also the correct evaluation of the slope parameter $h_{e}$ that, on its turn, depends both on the accuracy of the $q_{r}$ measurement and on that of $\Delta \theta_{r}$.

\section{Experimental setup}

An IR camera is mounted on a pan-tilt head and automatically driven by a suitable software to map the temperature of the inner walls of the insulated box on a refrigerated vehicle. Figure 2 shows the installed Pan-Tilt camera inside the truck. The angles of the Pan-Tilt camera are set as follows: for panning, from $0 \circ$ to $360^{\circ}$ with a step of $20^{\circ}$; for tilting, from 0 to $180^{\circ}$ with a step of $15^{\circ}$. Besides, on the upper-left of the one surface, a heat flux meter is set to measure the corresponding heat flux. The acquisition system used in this work is an infrared camera-FLIR A320, with a field of view (FOV) $25 \circ$ X19。. It records all the inner walls during the whole test. What's more, when the steady conditions are reached (a state period of time not less than 12 hours, according to the ATP standard [3]), several rounds of infrared images have been obtained. 
With standard ATP measurement during the test, a radiation heater is heating the inside the container to maintain a temperature about $32.5^{\circ} \mathrm{C}$. For outside, with the air circulating a velocity between 1 and 2 ms -1 in the tunnel, the temperature is maintained constant at about $7 \circ \mathrm{C}$. Thus makes a temperature difference between inside air and outside of $\Delta \theta=25.5^{\circ} \mathrm{C}$. Two hypothesis are proposed in this test: 1$)$ the heat exchange coefficients, inside $\left(h_{i}\right)$ and outside $\left(h_{e}\right)$ of the container are constant; 2$)$ the heat diffusion is mainly 1D. These hypothesis serve the computation of heat flux for each surface.

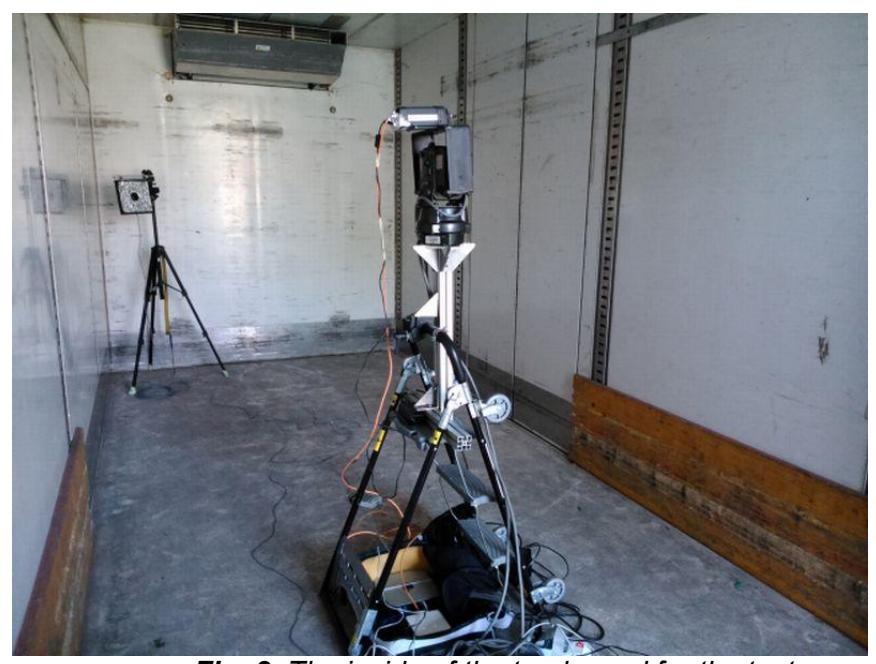

Fig. 2. The inside of the truck used for the test.

\section{Image processing}

Using a pan-tilt head often makes it easy to ensure a uniform coverage of the visual field with 360X180 full view, but also makes it possible to stitch the images using cylindrical or spherical coordinates and pure translation. Cylindrical and Spherical panoramas are commonly used because of their ease of construction. In our case, spherical panoramas is more convenient. Szeliski and Shum [11] explain in details how to compute approximate spherical projection using focal length. Brief conversion is shown in Figure 3. Therefore, the conclusion is that, given focal length $f$, image coordinates $(x$, $y)$, the corresponding spherical coordinates $\left(x^{\prime}, y^{\prime}\right)$ is:

$$
\begin{aligned}
& x^{\prime}=f \cdot \tan \left(\frac{x-x_{c}}{f}\right)+x_{c} \\
& y^{\prime}=f \cdot \frac{\tan \left(\frac{y-y_{c}}{f}\right)}{\cos \left(\frac{x-x_{c}}{f}\right)}+y_{c}
\end{aligned}
$$

where $\left(x_{c}, y_{c}\right)$ is the center coordinate of spherical image. 


\section{Spherical projection}

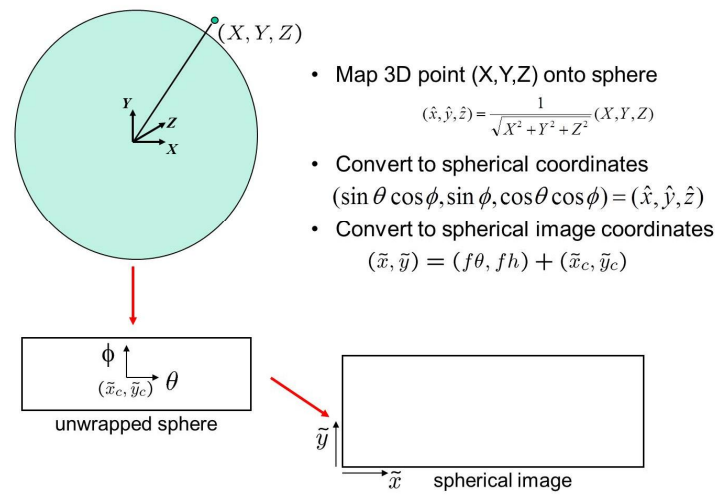

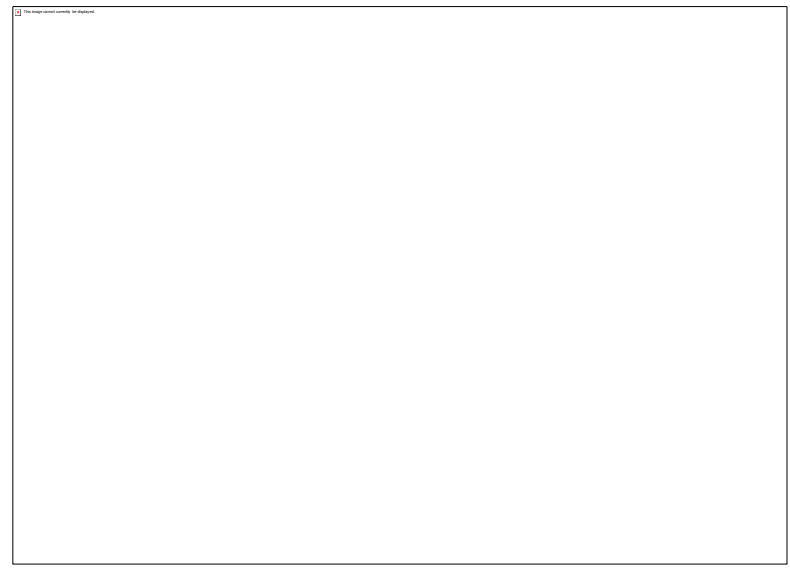

Fig. 3. Spherical projection.

Orginal image and its spherical projection are shown in Figure 4.
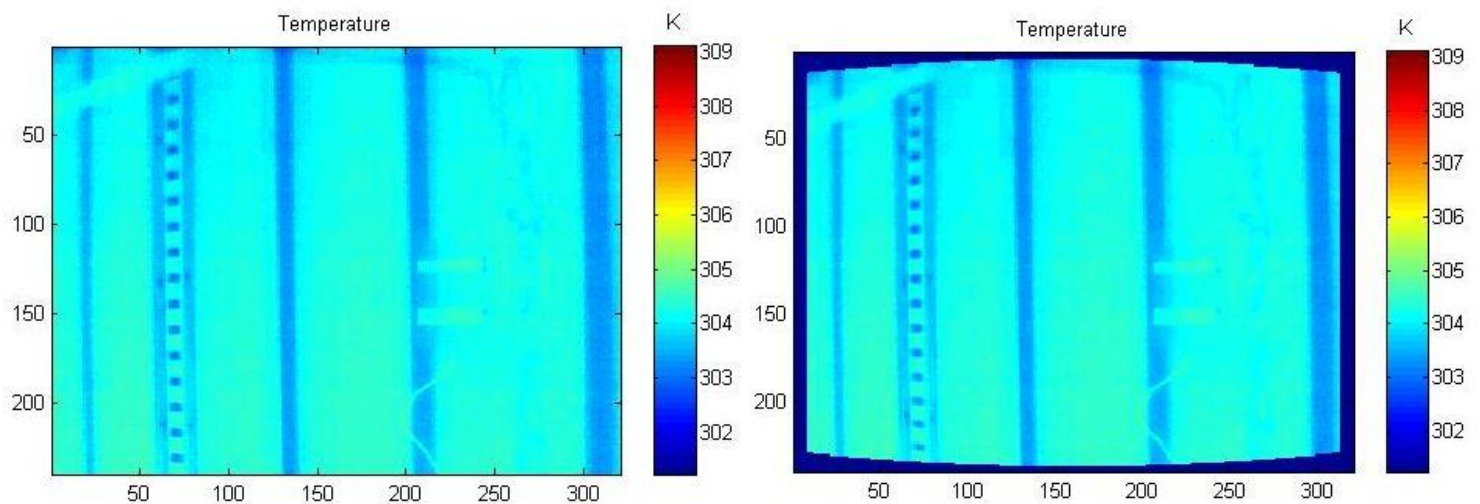

Fig. 4. Original IR image (left) and its spherical projection (right).

To estimate the translation between images, a scheme is elucidated in Figure 5, where $n$ is the width of each image, $\Delta n$ is the displacement for image translation, for a given Pan angle $\left(20^{\circ}\right)$ and a field of view $\left(25^{\circ} \times 19^{\circ}\right)$. Therefore $\Delta n$ can be obtained by a simple calculation:

$$
\Delta n=\frac{n}{2} \cdot\left[1-\tan \left(20^{\circ}-25^{\circ} / 2\right) \cdot \tan \left(90^{\circ}-25^{\circ} / 2\right)\right]
$$

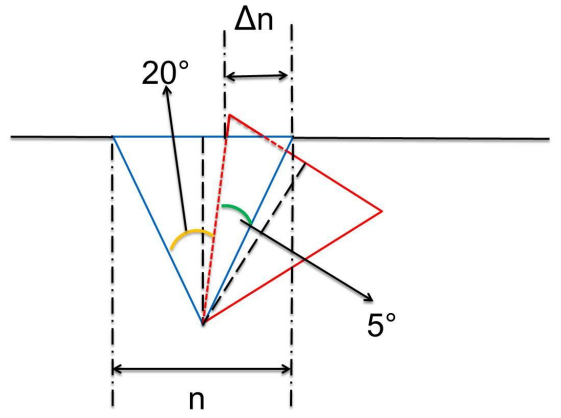

Fig. 5. Estimation of translation between images.

After estimating the translations, one starts to make images stitching. In this case, for the blended regions between images, one uses the max function of Matlab which returns the maximum value in the blended regions of two images. 
The reason is that after the spherical projection of original images, there are several regions with $\mathrm{NaN}$ values. Therefore one cannot make average from the two overlapping images. The iterate calculation is shown in Figure 6 and the result in the IR is shown in Figure 7.

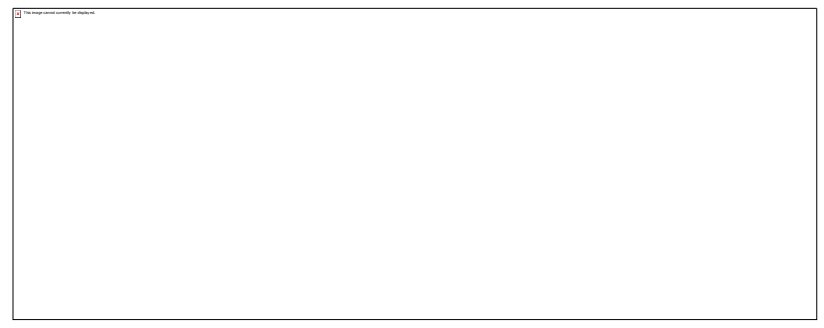

Fig. 6. Stitching of images.

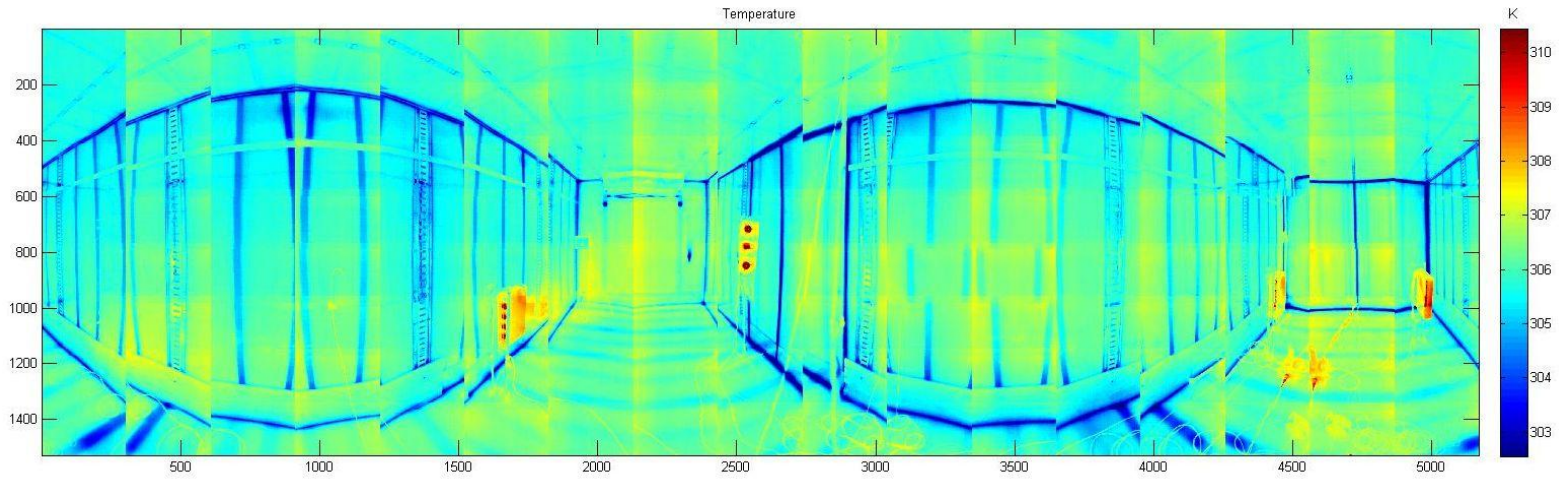

Fig. 7. Temperature panorama of the inside vehicle.

\section{Heat flux panorama}

According to equation (3) and (4) the auxiliary measurement of the heat flux in a reference point allows to transform the surface temperature image (panorama) in a heat flux image (panorama). In Figure 8 the measurement of the heat flux in the reference point is shown from the beginning of the test until the steady condition is reached. The panorama of Figure 7 is therefore transformed in the panorama of Figure 9 representing the map of heat flux in each pixel of the inner part of the insulated vehicle.

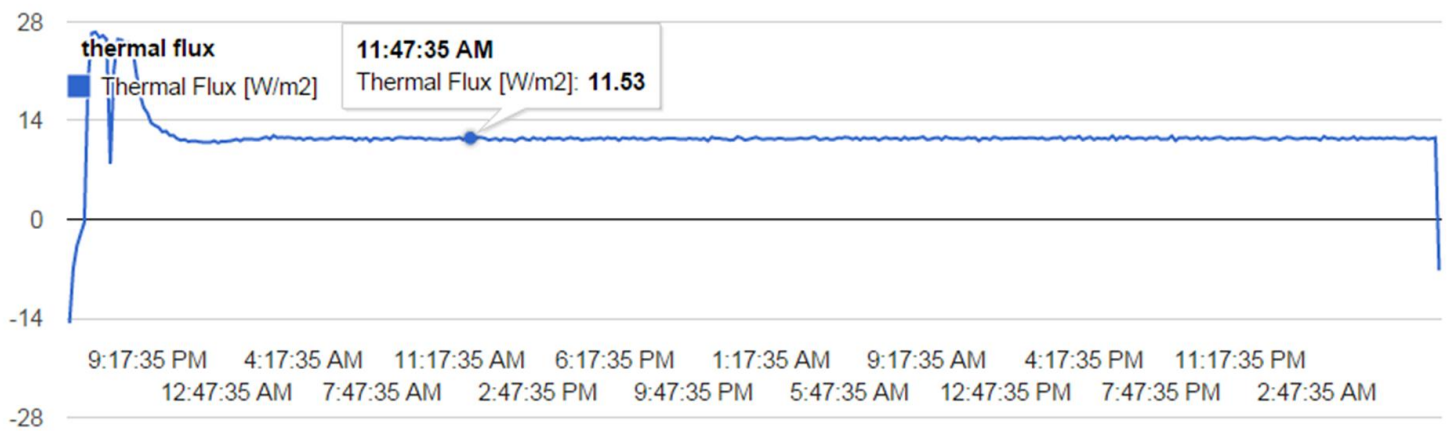

Fig. 8. Heat Flux meter measurement. 
Fig. 9. Heat Flux panorama of the inner part of the vehicle.

\section{Conclusion}

This work is mainly focused on mapping the heat flux on the internal surface of an insulated vehicle by Infrared thermography technique. The ATP standard measurement was performed to obtain the experimental results, meanwhile an IR camera is mounted on a pan-tilt head and automatically driven by a suitable software to map the temperature of the inner walls at the steady condition, in order to analyze and compute the corresponding heat flux on entire surfaces. A simple thermal resistance model has been applied to realize the computation. The final temperature figures showed a good uniform distribution, and several defects in the structure like thermal bridges or air leakages could be identified. A reference zone of the internal wall is measured by a thermal flux meter, then with that reference the whole surface heat flux map have been figured out. Besides, when all round of images are obtained, a panoramic view of the heat flux can be composed. Image processing techniques such as spherical projection, image translation and stitching have been performed with the raw images. The preliminary results demonstrate the algorithm can work, however the narrow view of the IR camera and the similarity of the internal surface of the vehicle make much difficult to create the full-view panorama by automatic detectors between images, which leads to a manual detection that took much more time to complete. Nonetheless, the full view of panorama can still be accomplished with more patience. Maybe some other better algorithm could come up with good suggestions.

\section{REFERENCES}

[1] ATP - UN-ECE, Agreement Transport Perishables. Agreement on the interna- tional carriage of perishable foodstuffs and on the special equipment to be used for such carriage, Geneva 1970.

[2] ATP(treaty). http://en.wikipedia.org/wiki/ATP_(treaty).

[3] S Rossi, P Bison, GP Cuogo, and E Grinzato. K-value estimation on refrigerated vehicles by thermographic analysis. SPIE Defense, Security, and Sensing, pages 72990N-72990N, 2009.

[4] Paolo G Bison, Chiara Bressan, Ermanno G Grinzato, and Sergio Marinetti. Au- tomatic air and surface temperature measure by ir thermography with perspec- tive correction. Applications in Optical Science and Engineering, pages 252-260, 1993.

[5] P Bison, A Bortolin, G Cadelano, G Ferrarini, K Furlan, and E Grinzato. Geo- metrical correction and photogrammetric approach in thermographic inspection of buildings. In 11 th International Conference on Quantitative InfraRed Ther- mography, 2012.

[6] Amedeo Dragano, Stefano Rossi, Paolo Bison, and Girolamo Panozzo. Exper- imental evaluation of the optical absorption coefficient of a sandwich panel for insulated vehicles. Quantitative InfraRed Thermography Journal, 6(2):207-223, 2009.

[7] E Grinzato, P Bison, G Cadelano, and F Peron. R-value estimation by local thermographic analysis. SPIE Defense, Security, and Sensing, pages $76610 \mathrm{H}-76610 \mathrm{H}, 2010$.

[8] E Grinzato, G Padovan, and G Panozzo. Quality test by thermographical anal- ysis: an estimation of k-value. International Institute of Refrigeration, Commis- sion D2, pages 73-83.

[9] E Grinzato and G Panozzo. Comparison of measurement of k-value by atp in- ternal heating method and by thermographic analysis. International Institute of Refrigeration, Commission, D1,D2,D3:183-190.

[10] Thermal resistance. http://en.wikipedia.org/wiki/Thermal_resistance.

[11] Richard Szeliski and Heung-Yeung Shum. Creating full view panoramic im- age mosaics and environment maps. In Proceedings of the 24th annual con- ference on Computer graphics and interactive techniques, pages 251-258. ACM Press/Addison-Wesley Publishing Co., 1997. 